\title{
THE BETTI GROUPS OF THE PRODUCT OF TWO NORMAL SPACES
}

\author{
C. E. CLARK
}

1. Introduction. Let $R_{1}$ and $R_{2}$ be homeomorphic to open sets of normal spaces. Furthermore, let each of $R_{1}$ and $R_{2}$ contain infinitely many points. For these spaces Alexandroff has defined inner Betti groups. ${ }^{1}$ In this paper the inner Betti groups of the topological product $R_{1} \otimes R_{2}$ are studied.

Let $\tilde{u}^{r} \in B^{r}\left(R_{1}\right)$, where $B^{r}$ denotes the $r$-dimensional inner cohomology group with the integers as coefficient domain, and let $\tilde{u}^{\rho} \in B^{\rho}\left(R_{2}\right)$. To these two elements there corresponds an element $\tilde{u}^{r} \times \tilde{u}^{\rho} \in B^{r+\rho}\left(R_{1} \times R_{2}\right)$. All such elements with $r+\rho=n$ generate a subgroup $B_{1}^{n}\left(R_{1} \times R_{2}\right) \subset B^{n}\left(R_{1} \times R_{2}\right)$. We characterize this subgroup (Theorem 3). In addition, we characterize the factor-group $B_{2}^{n}\left(R_{1} \times R_{2}\right)=B^{n}\left(R_{1} \times R_{2}\right) / B_{1}^{n}\left(R_{1} \times R_{2}\right)$ (Theorem 4). In doing so we show that if $\tilde{u}^{s} \in B^{s}\left(R_{1}\right)$ is of order $\tilde{e}^{s} \neq 0$, and if $\tilde{u}^{\sigma} \in B^{\sigma}\left(R_{2}\right)$ is of order $\tilde{e}^{\sigma} \neq 0$, then to these two elements there corresponds an element $\left(\tilde{u}^{s}, \tilde{u}^{\sigma}\right) \in B_{2}^{s+\sigma-1}\left(R_{1} \times R_{2}\right)$.

To prove these results we employ Alexandroff's second definition of the inner Betti groups which uses barycentric subdivisions of coverings. ${ }^{2}$ Furthermore, Freudenthal's simplicial division of the product of two simplexes is used. ${ }^{3}$

2. The groups $B_{i}^{n}\left(K^{a} \times K^{\alpha} \bmod C^{a \alpha}\right), i=1,2$. In this section we state without proof some facts about products of complexes which are consequences of [3]. Let $K^{a}$ and $K^{\alpha}$ be finite complexes with subcomplexes $C^{a}$ and $C^{\alpha}$, respectively. Let $B^{n}$ denote the $n$-dimensional integral cohomology group. To $u^{r} \in B^{r}\left(K^{a} \bmod C^{a}\right)$ and $u^{\rho} \in B^{\rho}\left(K^{\alpha} \bmod C^{\alpha}\right)$ there corresponds the product of these cohomology classes $u^{r} \times u^{\rho} \in B^{r+\rho}\left(K^{a} \times K^{\alpha} \bmod C^{a \alpha}\right)$. We define $B_{1}^{n}\left(K^{a} \times K^{\alpha} \bmod C^{a \alpha}\right)$ to be the subgroup of $B^{n}\left(K^{a} \times K^{\alpha} \bmod C^{a \alpha}\right)$ generated by-we could say consisting of -all $u^{r} \times u^{\rho}$ with $r+\rho=n$. Let $e^{r}$ and $e^{\rho}$ be the orders of $u^{r}$ and $u^{\rho}$, respectively, with the understanding that $e=0$ when $u$ is free. Let $(a, b)$ denote the greatest common divisor of $a$ and $b$ with the understanding that $(a, 0)=a$.

Received by the editors July 2, 1942 .

${ }^{1}$ See $[1,7.2]$ (Numbers in brackets refer to references at end of paper).

${ }^{2}$ See $[1,9.22$ and 9.4$]$.

3 See $[2]$. 
THEOREM 1. A complete set of relations for the generators $u^{r} \times u^{\rho}$ of $B_{1}^{n}\left(K^{a} \times K^{\alpha} \bmod C^{a \alpha}\right)$ is given by

$$
\left(e^{r}, e^{\rho}\right)\left(u^{r} \times u^{\rho}\right)=0
$$

and

$$
\sum p_{i} u_{i}^{r}+\sum q_{j} u_{j}^{\rho}=\sum p_{i} q_{j}\left(u_{i}^{r} \times u_{j}^{\rho}\right)
$$

where the p's and $q$ 's are integers.

We define $B_{2}^{n}\left(K^{a} \times K^{\alpha} \bmod C^{a \alpha}\right)=B^{n}\left(K^{a} \times K^{\alpha} \bmod C^{a \alpha}\right) / B_{1}^{n}$ $\left(K^{a} \times K^{\alpha} \bmod C^{a \alpha}\right)$. This factor-group will be characterized in a way suitable for our later discussion.

Let $u^{s} \in B^{s}\left(K^{a} \bmod C^{a}\right)$ be of order $e^{s} \neq 0$ and $u^{\sigma} \in B^{\sigma}\left(K^{\alpha} \bmod C^{\alpha}\right)$ be of order $e^{\sigma} \neq 0$. Throughout the paper the superscripts $s$ and $\sigma$ will be used only for elements of order different from 0 , while superscripts $r$ and $\rho$ indicate that no restriction is made on the orders of the elements. We shall associate with $u^{s}$ and $u^{\sigma}$ an element $\left(u^{s}, u^{\sigma}\right) \in B_{2}^{s+\sigma-1}\left(K^{a} \times K^{\alpha} \bmod C^{a \alpha}\right)$. To do so let $z^{s} \in u^{s}, \dot{f}^{s-1}=e^{s} z^{s}$ where the dot denotes the coboundary operator, $z^{\sigma} \in u^{\sigma}$, and $\dot{f}^{\sigma-1}=e^{\sigma} z^{\sigma}$. For any cocycle $z$ let $H(z)$ denote the cohomology class with $z \in H(z)$. We define

$$
H\left\{\frac{1}{\left(e^{s}, e^{\sigma}\right)}\left(f^{s-1} \times f^{\sigma-1}\right) \cdot\right\} \in\left(u^{s}, u^{\sigma}\right) .
$$

We can show that $\left(u^{s}, u^{\sigma}\right)$ is independent of the choice of $z$ 's and $f^{\prime}$ s.

TheOREM 2. The cosets $\left(u^{s}, u^{\sigma}\right), s+\sigma-1=n$, generate $B_{2}^{n}\left(K^{a} \times K^{\alpha}\right.$ $\left.\bmod C^{a \alpha}\right)$, and a complete set of relations for these generators consists of

$$
\left(e^{s}, e^{\sigma}\right)\left(u^{s}, u^{\sigma}\right)=0
$$

and

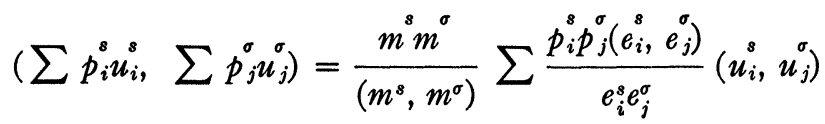

where we consider only those sums $\sum p_{i}^{s} u_{i}^{s}$ and $\sum p_{j}^{\sigma} u_{j}^{\sigma}$ with the following properties: the p's are integers, and the orders $m^{s}$ and $m^{\sigma}$ of $\sum p_{i}^{s} u_{i}^{s}$ and $\sum p_{j}^{\sigma} u_{j}^{\sigma}$ are the least common multiples of the orders of the terms $p_{i}^{s} u_{i}^{s}, i=1,2, \cdots$, and $p_{j}^{\sigma} u_{j}^{\sigma}, j=1,2, \cdots$, respectively.

3. Coverings and projections. We first associate with cofinal systems of coverings ${ }^{4}$ of $R_{1}$ and $R_{2}$ a cofinal system for $R_{1} \times R_{2}$ (our cover-

${ }^{4}$ See $[1,3.5]$. 
ings are finite and consist of open sets). With Alexandroff we say that a covering is multiplicative ${ }^{5}$ if the intersection of any two sets of the covering is also a set of the covering. Let $\left\{\Omega^{a}\right\}$ and $\left\{\Omega^{\alpha}\right\}$ be cofinal systems of multiplicative coverings of $R_{1}$ and $R_{2}$, respectively. Let $\Omega^{a \alpha}=\Omega^{a} \times \Omega^{\alpha}$. Let $\Omega^{b \beta}$ follow $\Omega^{a \alpha}$ if both $\Omega^{b}$ follows $\Omega^{a}$ and $\Omega^{\beta}$ follows $\Omega^{\alpha}$. It is easily seen that $\left\{\Omega^{a \alpha}\right\}$ is a cofinal system of multiplicative coverings of $R_{1} \times R_{2}$.

If $\Omega^{b}$ follows $\Omega^{a}$, let $S_{a}^{b}$ be the canonical projection ${ }^{6}$ of $\Omega^{b}$ into $\Omega^{a}$; that is, if $M^{b} \in \Omega^{b}$, then $S_{a}^{b} M^{b}$ is the smallest open set of $\Omega^{a}$ such that $M^{b} \supset S_{a}^{b} M^{b}$. Similarly we have the canonical projection $S_{\alpha}^{\beta}$ of $\Omega^{\beta}$ into $\Omega^{\alpha}$ if $\Omega^{\beta}$ follows $\Omega^{\alpha}$. With these two projections we shall associate the canonical projection $S_{a \alpha}^{b \beta}$ of $\Omega^{b \beta}$ into $\Omega^{a \alpha}$. Let $M^{b} \in \Omega^{b}$ and $M^{\beta} \in \Omega^{\beta}$. It is easily seen that

$$
S_{a \alpha}^{b \beta} M^{b} \times M^{\beta}=S_{a}^{b} M^{b} \times S_{\alpha}^{\beta} M^{\beta} .
$$

By the barycentric subdivision of a covering ${ }^{7}$ is meant that subcomplex of the nerve of the covering made up of the simplexes whose vertices are associated with decreasing sequences of open sets of the covering. Let $K^{a}, K^{\alpha}$, and $K^{a \alpha}$ be the barycentric subdivisions of $\Omega^{a}$, $\Omega^{\alpha}$, and $\Omega^{a \alpha}$. We shall show that $K^{a \alpha}$ is a simplicial subdivision of $K^{a} \times K^{\alpha}$.

First, let the $r$-simplex $t^{r}$ of $K^{a}$ be associated with the following sets of $\Omega^{a}$ :

$$
M_{0}^{a} \supset \cdots \supset M_{r}^{a}
$$

Similarly, let $t^{\rho}$ of $K^{\alpha}$ be associated with

$$
M_{0}^{\alpha} \supset \cdots \supset M_{\rho}^{\alpha}
$$

Corresponding to these two simplexes there is a set of $(r+\rho)$-simplexes of $K^{a \alpha}$ in (1-1)-correspondence with the sequences

(9) $\quad M_{0}^{a} \times M_{0}^{\alpha} \supset \cdots \supset M_{i}^{a} \times M_{j}^{\alpha} \supset M_{k}^{a} \times M_{l}^{\alpha} \supset \cdots \supset M_{r}^{a} \times M_{\rho}^{\alpha}$

where $k \geqq i, l \geqq j$, and $k+l=i+j+1$. But Freudenthal has shown ${ }^{8}$ that this set of simplexes forms a simplicial division of $t^{r} \times t^{\rho}$. Furthermore, the cells $t^{r} \times t^{\rho}$ determined by (9) form a cell complex that is isomorphic with $K^{a} \times K^{\alpha}$.

5 See $[1,9.2]$.

${ }^{6}$ See $[1,9.3]$.

${ }^{7}$ See $[1,9.22]$.

${ }^{8}$ See $[2]$. 
Each of the complexes $K^{a}, K^{\alpha}$, and $K^{a \alpha}$ has a special subcomplex ${ }^{9}$ $C^{a}, C^{\alpha}$, and $C^{a \alpha}$ made up of the simplexes all of whose vertices are associated with open sets whose closures are not bicompact. We shall show that $C^{a} \times K^{\alpha}+K^{a} \times C^{\alpha} \subset C^{a \alpha}$. Indeed, suppose the closure of each set of (7) is not bicompact. Then the closure of each set of any sequence of (9) is not bicompact (because the continuous image of a bicompact set is bicompact, and a factor of a product of two sets is a continuous image of the product). The same argument holds for a simplex of $C^{\alpha}$, and the above inclusion holds.

Next suppose $t^{r}$ is not in $C^{a}$. This means that $\bar{M}_{r}^{a}$, the closure of the final term of (7), is bicompact. Also suppose $t^{\rho}$ is not in $C^{\alpha}$. This means that $\bar{M}_{\rho}^{\alpha}$, from (8), is bicompact. Since the product of the closures of two sets is the closure of the product, and since the product of two bicompact sets is bicompact, the last term of (9) has a bicompact closure. This means that $t^{r} \times t^{\rho}$ is not in $C^{a \alpha}$. This with the above inclusion means that $C^{a} \times K^{\alpha}+K^{a} \times C^{\alpha}=C^{a \alpha}$. We have proved this lemma.

Lemma. The complex $K^{a \alpha}$ is a simplicial division of $K^{a} \times K^{\alpha}$, and $C^{a \alpha}$ is the division of $C^{a} \times K^{\alpha}+K^{a} \times C^{\alpha}$.

The canonical projections $S_{a}^{b}, S_{\alpha}^{\beta}$, and $S_{a \alpha}^{b \beta}$ determine the usual homomorphic mappings $\rho_{a}^{b}, \rho_{\alpha}^{\beta}$, and $\rho_{a \alpha}^{b \beta}$ of the chains of $K^{b} \bmod C^{b}$, and so on, into the chains of $K^{a} \bmod C^{a}$, and so on. ${ }^{10} \mathrm{We}$ shall show that

$$
\rho_{a \alpha}^{b \beta}\left(t^{r} \times t^{\rho}\right)=\rho_{a}^{b} t^{r} \times \rho_{\alpha}^{\beta} t^{\rho} .
$$

In (7) replace the superscript $a$ by $b$ and let the resulting sequence denote the open sets associated with $t^{r}$. In the same way we replace $\alpha$ by $\beta$ in (8) and (9) to obtain sequences associated with $t^{\rho}$ and $t^{r} \times t^{p}$. From (6) and (9) we see that the left side of (10) is associated with the sequences $S_{a}^{b} M_{0}^{b} \times S_{\alpha}^{\beta} M_{0}^{\beta} \supset \cdots$ But these sequences are associated with $\rho_{a}^{b} t^{r} \times \rho_{\alpha}^{\beta} t^{\rho}$ because $\rho_{a}^{b} t^{r}$ is associated with $S_{a}^{b} M_{0}^{b} \supset \ldots$ and $\rho_{\alpha}^{\beta} t^{\rho}$ is associated with $S_{\alpha}^{\beta} M_{0}^{\beta} \supset \cdots$.

The canonical projections $S_{a}^{b}, S_{\alpha}^{\beta}$, and $S_{a \alpha}^{b \beta}$ determine the usual homomorphic mappings $\sigma_{b}^{a}, \sigma_{\beta}^{\alpha}$, and $\sigma_{b \beta}^{a \alpha}$ of the chains of $K^{a} \bmod C^{a}$, and so on, into the chains of $K^{b} \bmod C^{b}$, and so on. ${ }^{10}$ From (10) we have

$$
\left.\underset{\sigma_{b \beta}\left(f^{a}\right.}{a} \times f^{\alpha}\right)=\underset{\sigma_{b} f^{a}}{a} \times \underset{\sigma_{\beta} f^{\alpha}}{\alpha} .
$$

Furthermore, these $\sigma$ 's determine homomorphic mappings $\pi_{b}^{a}$ of

${ }^{9}$ See $[1,9.1]$.

${ }^{10}$ See $[1,2.2]$. 
$B^{n}\left(K^{a} \bmod C^{a}\right)$ into $B^{n}\left(K^{b} \bmod C^{b}\right), \pi_{\beta}^{\alpha}$ of $B^{n}\left(K^{\alpha} \bmod C^{\alpha}\right)$ into $B^{n}\left(K^{\beta} \bmod C^{\beta}\right)$, and in view of the lemma $\pi_{b \beta}^{a \alpha}$ of $B^{n}\left(K^{a} \times K^{\alpha} \bmod C^{a \alpha}\right)$ into $B^{n}\left(K^{b} \times K^{\beta} \bmod C^{b \beta}\right) .{ }^{11}$ From (11) we have

$$
\pi_{b \beta}^{a \alpha}\left(u^{a} \times u^{\alpha}\right)=\pi_{b}^{a} u^{a} \times \underset{\pi_{\beta}^{\alpha} u^{\alpha}}{\alpha} .
$$

4. The inner Betti groups of $R_{1} \times R_{2}$. Let $B^{n}\left(R_{1}\right), B^{n}\left(R_{2}\right)$, and $B^{n}\left(R_{1} \times R_{2}\right)$ be the inner Betti groups defined in $\S 1$. Let $\tilde{u}^{r} \in B^{r}\left(R_{1}\right)$ be of order $\tilde{e}^{r}$, and $\tilde{u}^{\rho} \in B^{\rho}\left(R_{2}\right)$ be of order $\tilde{e}^{\rho}$. We shall define $\tilde{u}^{r} \times \tilde{u}^{\rho} \in B^{r+\rho}\left(R_{1} \times R_{2}\right)$. Let $u^{r a} \in \tilde{u}^{r}$ and $u^{\rho \alpha} \in \tilde{u}^{\rho}$ where $a$ and $\alpha$ are indices of coverings. From (12) we have $\pi_{b \beta}^{a \alpha}\left(u^{r a} \times u^{\rho \alpha}\right)=\pi_{b}^{a} u^{r a} \times \pi_{\beta}^{\alpha} u^{\rho \alpha}$. Hence we can define

$$
u^{r a} \times u^{\rho \alpha} \in \tilde{u}^{r} \times \tilde{u}^{\rho}
$$

and determine a bundle independent of the choice of coverings.

A consequence of (2) is

$$
\sum p_{i} \tilde{u}_{i}^{r} \times \sum q_{j} \tilde{u}_{j}^{p}=\sum p_{i} q_{j}\left(\tilde{u}_{i}^{r} \times \tilde{u}_{j}^{p}\right) .
$$

It is possible to find coverings with indices $b$ and $\beta$ so that $u^{r b}$ and $u^{\rho \beta}$ are of order $\widetilde{e}^{r}$ and $\widetilde{e}^{\rho}$, respectively, (indeed, $\tilde{e}^{r} u^{r a}$ is in the zero bundle, and any cohomology class of the zero bundle can be projected into a zero cohomology class; furthermore, $u^{r b}$ cannot be of order less than $\widetilde{e}^{r}$ ). This with (1) gives

$$
\left(\widetilde{e}^{r}, \widetilde{e}^{\rho}\right)\left(\tilde{u}^{r} \times \tilde{u}^{\rho}\right)=0 .
$$

Let $B_{1}^{n}\left(R_{1} \times R_{2}\right)$ be the subgroup of $B^{n}\left(R_{1} \times R_{2}\right)$ generated by the bundles (13) with $r+\rho=n$.

THEOREM 3. Relations (14) and (15) are a complete set for the generators (13) of $B_{1}^{n}\left(R_{1} \times R_{2}\right)$.

Proof. Let the finite sum $\sum p_{i j} \tilde{u}_{i}^{r} \times \tilde{u}_{j}^{\rho}=0, r+\rho=n$, but $r$ and $\rho$ can vary from term to term. For some coverings with indices $a$ and $\alpha$ we have $\sum p_{i j}\left(u^{r a} \times u^{\rho \alpha}\right)=0$. Theorem 1 proves that this latter relation is a consequence of (1) and (2). Hence the original relation is a consequence of (14) and (15) q.e.d.

Equation (12) shows that $\pi_{b \beta}^{a \alpha} B_{1}^{n}\left(K^{a} \times K^{\alpha} \bmod C^{a \alpha}\right) \subset B_{1}^{n}\left(K^{b} \times K^{\beta}\right.$ $\left.\bmod C^{b \beta}\right)$. Hence there is a natural homomorphism $\Pi_{b \beta}^{a \alpha}$ of $B_{2}^{n}\left(K^{a} \times K^{\alpha} \bmod C^{a \alpha}\right)$ into $B_{2}^{n}\left(K^{b} \times K^{\beta} \bmod C^{b \beta}\right)$. This natural homomorphism is defined as follows. If $\phi^{a \alpha} u^{a \alpha}$ is the coset of $B_{2}^{n}\left(K^{a} \times K^{\alpha} \bmod C^{a \alpha}\right)$ that contains the cohomology class $u^{a \alpha}$ $\in B^{n}\left(K^{a} \times K^{\alpha} \bmod C^{a \alpha}\right)$, then

${ }^{11}$ See $[1,2.33]$. 


$$
\Pi_{b \beta \phi}^{a \alpha \phi^{a \alpha}} u^{a \alpha}=\phi^{b \beta} \pi_{b \beta}^{a \alpha} u^{a \alpha}
$$

We shall show that $\Pi_{c \gamma}^{b \beta} \Pi_{b \beta}^{a \alpha}=\Pi_{c \gamma}^{a \alpha}$. Indeed, using (16) and the fact that $\pi_{c \gamma}^{b \beta} \pi_{b \beta}^{a \alpha}=\pi_{c \gamma}^{a \alpha}$, we have $\Pi_{c \gamma}^{a \alpha} \phi^{a \alpha} u^{a \alpha}=\phi^{c \gamma} \pi_{c \gamma}^{a \alpha} u^{a \alpha}=\phi^{c \gamma} \pi_{c \gamma}^{b \beta} \pi_{b \beta}^{a \alpha} u^{a \alpha}=\Pi_{c \gamma}^{b \beta} \phi^{b \beta} \pi_{b \beta}^{a \alpha} u^{a \alpha}$ $=\Pi_{c \gamma}^{b \beta} \Pi_{b \beta}^{a \alpha} \phi^{a \alpha} u^{a \alpha}$ proving the equality in question. We have shown that $\left[B_{2}^{n}\left(K^{a} \times K^{\alpha} \bmod C^{a \alpha}\right) ; \Pi_{b \beta}^{\alpha \alpha}\right]$ is a spectrum. Let the direct limit of this spectrum be denoted by $B_{2}^{n}\left(R_{1} \times R_{2}\right)$.

Let $\tilde{u}^{s} \in B^{s}\left(R_{1}\right)$ and $\tilde{u}^{\sigma} \in B^{\sigma}\left(R_{2}\right)$ have orders $\tilde{e}^{s} \neq 0$ and $\tilde{e}^{\sigma} \neq 0$. We shall associate with these two bundles an element $\left(\tilde{u}^{s}, \tilde{u}^{\sigma}\right)$ $\in B_{2}^{s+\sigma-1}\left(R_{1} \times R_{2}\right)$. Consider a cohomology class of the coset $\left(u^{s a}, u^{\sigma \alpha}\right)$, $u^{s a} \in \tilde{u}^{s}, u^{\sigma \alpha} \in \tilde{u}^{\sigma}, u^{s a}$ of order $\tilde{e}^{s}$, and $u^{\sigma \alpha}$ of order $\tilde{e}^{\sigma}$. In view of (3) this cohomology class contains a cocycle which may be written

$$
\frac{1}{\left(\tilde{e}^{s}, \tilde{e}^{\sigma}\right)}\left(f^{s-1 a} \times f^{\sigma-1 \alpha}\right) \text {. }
$$

with $\tilde{f}^{s-1 a}=\widetilde{e}^{\delta} z^{s a}, z^{s a} \in u^{s a}$, and similar relations with superscript $\sigma$. We have

$$
\sigma_{b \beta}^{a \alpha}(17)=\frac{1}{\left(\tilde{e}^{s}, \tilde{e}^{\sigma}\right)}\left(\sigma_{b}^{a} f^{s-1 a} \times \sigma_{\beta}^{\alpha} f^{\sigma-1 \alpha}\right) .
$$

because of (11) and the fact that the operation of forming coboundary commutes with $\sigma_{b \beta}^{a \alpha}{ }^{12}$ But $\left(\sigma_{b}^{a} f^{s-1 a}\right)^{\cdot}=\tilde{e}^{s} \sigma_{b}^{a} z^{s a}$ with the same relation for superscript $\sigma$. Hence (18) is in a cohomology class of the coset $\left(\pi_{b}^{a} u^{s a}, \pi_{\beta}^{\alpha} u^{\sigma \alpha}\right)$. Letting $H(z)$ still denote the cohomology class that contains the cocycle $z$, we have using these results and (16) that $\Pi_{b \beta}^{a \alpha}\left(u^{s a}, u^{\sigma \alpha}\right)=\Pi_{b \beta}^{a \alpha} \phi^{a \alpha} H(17)=\phi^{b \beta} \pi_{b \beta}^{a \alpha} H(17)=\phi^{b \beta} H(18)=\left(\pi_{b}^{a} u^{s a}, \pi_{\beta}^{\alpha} u^{\sigma \alpha}\right)$. Hence we have a unique bundle $\left(\tilde{u}^{s}, \tilde{u}^{\sigma}\right) \in B_{2}^{s+\sigma-1}\left(R_{1} \times R_{2}\right)$ defined by

$$
\left(u^{s a}, u^{\sigma \alpha}\right) \in\left(\tilde{u}^{s}, \tilde{u}^{\sigma}\right) .
$$

From (4) we obtain

$$
\left(\tilde{e}^{s}, \tilde{e}^{\sigma}\right)\left(\tilde{u}^{s}, \tilde{u}^{\sigma}\right)=0 .
$$

Furthermore, let the sums $\sum p_{i}^{s} \tilde{u}_{i}^{s}$ and $\sum p_{j}^{\sigma} \tilde{u}_{j}^{\sigma}$ satisfy the conditions on the sums in (5). From (5) we have a relation which we label (21). The relation (21) is obtained from (5) by replacing each $u$ by $\tilde{u}$.

TheOREM 4. The elements $\left(\tilde{u}^{s}, \tilde{u}^{\sigma}\right), s+\sigma-1=n$, generate $B_{2}^{n}\left(R_{1} \times R_{2}\right)$, and relations (20) and (21) are a complete set for these generators.

Theorem 4 is a consequence of Theorem 2 and the definition of

${ }^{12}$ See [1, Theorem 2.221]. 
$B_{2}^{n}\left(R_{1} \times R_{2}\right)$ as a direct limit. The proof is similar to the proof of Theorem 3 .

Theorem 5. We have $B_{2}^{n}\left(R_{1} \times R_{2}\right)=B^{n}\left(R_{1} \times R_{2}\right) / B_{1}^{n}\left(R_{1} \times R_{2}\right)$.

Proof. It follows from (16) that there is a homomorphic mapping of $B^{n}\left(R_{1} \times R_{2}\right)$ upon $B_{2}^{n}\left(R_{1} \times R_{2}\right) .{ }^{13}$ The kernel of this homomorphism consists of the bundles of $B^{n}\left(R_{1} \times R_{2}\right)$ which contain elements of the groups $B_{1}^{n}\left(K^{a} \times K^{\alpha} \bmod C^{a \alpha}\right){ }^{13}$ From (12) and (18) we see that this kernel is precisely $B_{1}^{n}\left(R_{1} \times R_{2}\right)$.

\section{REFERENCES}

1. P. Alexandroff, General combinatorial topology, Trans. Amer. Math. Soc. vol. 49 (1941) pp. 41-105.

2. H. Freudenthal, Eine Simplizialzerlegung des Cartesischen Productes Zweier Simplexe, Fund. Math. vol. 29 (1937) pp. 138-144.

3. S. Lefschetz, Algebraic topology, Amer. Math. Soc. Colloquium Publications vol. 27 (1942) pp. 139-143.

Purdue University

${ }^{13}$ See [1, Theorems 3.41 and 3.411]. 